Clinical Medicine \& Research

\title{
Review
}

\section{Preventing Surgical Site Infections}

Ronald Lee Nichols, MD, Department of Surgery, Tulane University Health Sciences Center School of Medicine, New Orleans, Louisiana

\begin{abstract}
Over the past 50 years, increased interest in the discipline of surgical infection has resulted in advances in post-surgical infection control. Early investigations focused on the importance of anaerobic microflora to postoperative infection and paved the way for significant improvements in prophylactic and therapeutic antibiotic treatment of surgical patients. Later research centered on the identification of risk factors to better predict postoperative infection rates. This article reviews the evolution of postoperative infection control and highlights antibiotic prophylaxis in specific clinical situations.
\end{abstract}

\section{INTRODUCTION}

When one looks retrospectively, the mid-1960s appear to represent the pivotal time period in which clinical and experimental interests and investigations in the area of surgical infections began to emerge. Up to this time, only a few surgeons, including Altemeier, Beck, Burke, Dineen, Laufman and their colleagues, had demonstrated interest and expertise in this slowly developing surgical discipline. However, most clinical and laboratory work was carried out by microbiologists or medical infectious disease specialists. Studies published in the early 1970s concerning the qualitative and quantitative nature of the various human microflora in health and disease helped unravel the mystery of the importance of the predominant anaerobic microflora to postoperative infection. ${ }^{1}$ This knowledge led to significant improvements in the use of prophylactic and therapeutic antibiotic regimens in the surgical patient that were largely published in the late 1970s and early 1980s. Innovative strategies by pharmaceutical companies provided many new antibiotic agents and classes of agents.

Attention to the general and procedure-specific patient risk factors for the development of postoperative surgical site infection started to surface in the mid 1980s and were more clearly understood by the mid 1990s. This helped clinicians identify high and low risk surgical patients within the historical classes of operative procedures, allowing them to better predict the expected postoperative surgical site infection rates in individual patients. Investigations concerning the value of alterations in therapy in the different risk groups began in the mid-1990s and continued into the $21^{\text {st }}$ century.

RECEIVED: OCTOBER 23, 2003

REPRINT REQUESTS:

Ronald Lee Nichols, MD

Department of Surgery SL22

Tulane University Health Sciences Center

School of Medicine

1430 Tulane Avenue

New Orleans, LA 70112-2699

Telephone: 504-588-5168

Fax: 504-586-3843

Email: ronald.nichols@tulane.edu
REVISED AND ACCEPTED: JANUARY 10, 2004

\section{KEYWORDS:}

Surgical wound infection; Anti-bacterial agents; Antibiotic prophylaxis;

Infection control; Risk factors 


\section{PREDICTING WOUND SITE INFECTIONS}

Until the late 1980s most infection control officers, operating room nurses, and surgeons thought that the type of operative procedure undertaken was the most critical factor in predicting the postoperative surgical site infection rate. The often-quoted infection rates for the traditional classification of operative procedures were as follows: clean $(<2 \%)$, clean-contaminated ( $5 \%$ to $15 \%)$, contaminated (15\% to $30 \%)$, and dirty $(>30 \%)$.

Haley and colleagues ${ }^{2}$ were the first to publish on the importance of identifying individual patients who are at high risk of surgical site infection in each category of operative procedure with the hope that the approach would result in an increase in the efficiency of routine surgical site infection surveillance and control. Analyzing 10 possible risk factors by step-wise multiple logistic regression techniques, a model was developed containing four risk factors (abdominal operations, operations lasting longer than 2 hours, contaminated or dirty-infected operation by the traditional wound classification system, and patients having three or more different diagnoses), and utilized the resultant formula to predict an individual patient's probability of developing a postoperative surgical site infection. This approach was then tested on another group of 59,352 surgical patients admitted from 1975 to 1976 and was found to be a valid predictor of surgical site infection.

Haley and colleagues concluded that their simplified index predicted surgical site infection risk approximately twice as well as the traditional classification of wound contamination. Utilizing this model, low-, medium-, and high-risk levels of developing surgical site infection were identified in each of the categories of the traditional wound classification system. The overall surgical site infection rate in this study did progressively increase from clean $(2.9 \%)$, to clean-contaminated $(3.9 \%)$, to contaminated $(8.5 \%)$, to dirty-infected (12.6\%). However, a wide range of infection risk in patients in each category was noted in clean operations, $1.1 \%$ (low risk) to $15.8 \%$ (high risk); in clean-contaminated operations, $0.6 \%$ (low risk) to $17.7 \%$ (high risk); in contaminated operations, $4.5 \%$ (medium risk) to $23.9 \%$ (high risk); and in dirty-infected operations, $6.7 \%$ (medium risk) to $27.4 \%$ (high risk). It should be noted that no low-risk category patients were identified in contaminated and dirty-infected operations.

Following the work of Haley and colleagues, investigators at the Centers for Disease Control and Prevention (CDC) reported on a composite risk index used in the National Nosocomial Infections Surveillance (NNIS) System. ${ }^{3}$ This risk index was based on a modification of the one developed in the Study on the Efficacy of Nosocomial Infection Control (SENIC) project. The NNIS risk index uses the traditional wound classification system but attempts to improve on the SENIC index in several ways. First, instead of utilizing three discharge diagnoses to identify host factors as a risk of infection, the NNIS risk index uses a dichotomization of the American Society of Anesthesiology score. Its ease for collecting data and its objectivity seem advantageous. Second, the NNIS risk index uses a procedure-related cut point to indicate a long duration of surgery for an individual procedure, rather than a 2-hour cut point for all procedures. For some procedures (e.g., cesarean section), the cut point is 1 hour. For other procedures (e.g., coronary artery bypass graft operation), the cut point is 5 hours. This procedure-related duration of surgery cut point provides the NNIS risk index with additional discriminating power for specific operative procedures.

\section{PREVENTION OF SURGICAL SITE INFECTIONS}

The most critical factors in the prevention of postoperative infections, although difficult to quantify, are the sound judgment and proper technique of the surgeon and surgical team, as well as the general health and disease state of the patient. ${ }^{4}$ Other factors influence the development of postoperative surgical site infection, especially in clean surgical procedures for which the infection rate $(<3 \%)$ is related to airborne exogenous microorganisms.

In 1999, the CDC's Health Care Infection Control Practices Advisory Committee published revised guidelines for the prevention of infections. These guidelines delve extensively into the literature concerning perioperative factors associated with postoperative infections. ${ }^{5}$ Many factors proven to influence surgical site infections, such as length of preoperative stay, duration of operation, preoperative cleansing and shaving techniques, use of abdominal drains, presence of remote infection, and adequate control of serum glucose levels perioperatively are among others authoritatively reviewed. Lacking are studies that offer no recommendations (unresolved issue) on such factors as preoperative application of mupirocin to the nares, enhancement of nutritional support, and enhancement of wound space oxygenation. The subject of operating room or patient temperature is not considered in these CDC guidelines.

\section{PROPHYLACTIC ANTIBIOTIC USAGE IN THE SURGICAL PATIENT}

The use of antibiotic prophylaxis before surgery has evolved greatly in the last 30 years. Improvements in the timing of initial administration, the appropriate choice of antibiotic agents, and shorter durations of administration have more clearly defined the value of this technique in reducing postoperative surgical site infections.

The choice of parenteral prophylactic antibiotic agents and the timing and route of administration have become standardized on the basis of well-planned prospective clinical studies. In elective, clean surgical procedures using a foreign body and in clean-contaminated procedures, it is generally recommended that anesthesia personnel administer intravenously a single dose of cephalosporin (e.g. cefazolin) 
just before incision. ${ }^{6}$ Additional doses are generally recommended only when the operation lasts longer than 2 to 3 hours. Controversial areas include the routine use of antibiotic prophylaxis in clean surgical procedures, such as hernia repair or breast surgery. This subject has been summarized in a published review. ${ }^{6}$ Specific clinical situations and other approaches to antibiotic prophylaxis are reviewed below.

\section{Antibiotic Prophylaxis Before Elective Colon Resection}

The human colon and distal small intestine contain an enormous reservoir of facultative and anaerobic bacteria separated from the rest of the body by the mucous membrane. A reliable method of sterilizing the colonic contents has been a goal of surgeons throughout the last century. In the past 25 years, clinical trials have demonstrated that to substantially reduce septic complications after elective colon surgery, antibiotics must have activity against both colonic aerobes (e.g., Escherichia coli) and anaerobes (e.g., Bacteroides fragilis). This finding was first reported over 25 years ago. ${ }^{7}$ Today, approaches to mechanical cleansing differ widely. Modern approaches include standard outpatient mechanical cleansing with dietary restriction, cathartics, and enemas for a 2-day period, or whole-gut lavage with polyethylene glycol, or the use of oral sodium phosphate solution done the day before the operation.

Most surgeons use both antibiotics and mechanical cleansing for preoperative preparation before elective colon resection. 8,9 Three separate regimens of oral agents combine neomycin with erythromycin base, metronidazole, or tetracycline. The most popular regimen in the United States has been the neomycin-erythromycin base preparation, introduced in 1972.10

In a survey published in $1997,58 \%$ of board-certified colorectal surgeons described their bowel preparation practices before elective procedures. ${ }^{8}$ All respondents used mechanical cleansing preparations, either oral polyethylene glycol solution $(70.9 \%)$ or oral sodium phosphate solution with or without bisacodyl (28.4\%), and accepted methods of dietary restriction, cathartics, and enemas (28.4\%). Most of the responding surgeons $(86.5 \%)$ added both oral and parenteral antibiotics to the regimen, while $11.5 \%$ added only parenteral antibiotics, $1.1 \%$ added only oral antibiotics, and $0.9 \%$ did not add antibiotics. Oral neomycin and erythromycin or metronidazole were combined with a perioperative parenteral antibiotic by $77.8 \%$ of respondents. Most patients started the preparation as outpatients the day before surgery, and the parenteral drugs were added to the regimen 1 to 2 hours before the procedure. The use of outpatient bowel preparation is increasing. However, patient selection is critical, and education is needed to reduce the rate of complications.

\section{Antibiotic Prophylaxis for Appendectomy}

The pathologic state of the appendix is the most important determinant of postoperative infection. ${ }^{11}$ Wound infection after appendectomy for perforative or gangrenous appendicitis is four to five times higher than that for early disease. A prospective study of non-perforated appendicitis, using a logistic regression analysis of risk factors, showed that the risk for postoperative infection is related to lack of perioperative antibiotic prophylaxis and to the determination that the appendix was gangrenous. ${ }^{11}$ Because the pathologic state of the appendix often cannot be determined before or during operation, a parenteral antibiotic agent is recommended as prophylaxis in all patients.

Regimens with activity against both facultative gram-negative bacilli and anaerobes are more effective than those active only against the aerobes. The use of antimicrobial agents in perforated appendicitis with evidence of local or generalized peritonitis, intra-abdominal abscess, or both should be considered therapeutic rather than prophylactic.

\section{Preventive Antibiotics in Penetrating Abdominal Trauma} Hollow-lumen visceral damage with associated escape of endogenous microorganisms is the main risk factor for postoperative infections after exploratory laparotomy for penetrating abdominal trauma. A single dose of parenterally administered antibiotic, given just before abdominal exploration for penetrating abdominal trauma, is associated with a low postoperative infection rate in patients with no observed gastrointestinal leakage. ${ }^{12}$ If gastrointestinal leakage is identified at the time of the operation, continuing the antibiotic agents for 1 to 3 days is usually recommended. It is important to use antibiotic agents with both facultative and anaerobic activity. Leaving the operative wound open and packed with saline-soaked gauze decreases the incidence of postoperative wound infection in patients at high risk. ${ }^{13}$

\section{Preventive Antibiotic Usage in Traumatic Chest Injuries}

Recently published studies have shown the value of parenteral antibiotic prophylaxis in the prevention of pneumonia or empyema after the placement of a chest tube to correct the hemopneumothorax associated with chest trauma. ${ }^{14,15}$ In one study, $500 \mathrm{mg}$ of cefazolin was given intravenously every 8 hours for 24 hours. ${ }^{14}$ In the other study, $1 \mathrm{~g}$ of cefonicid was administered every 24 hours until the chest tube was removed, usually before 5 days. ${ }^{15}$ In both studies patients receiving antibiotics had substantially lower infection rates than those receiving placebos.

\section{CONCLUSION}

Recent improvements in antibiotic prophylaxis, including the timing of initial administration, appropriate choice of antibiotic agents, and shortening the duration of administration have established the value of this technique in many clinical surgical settings. A single dose systemic regimen of an appropriately chosen cephalosporin given during the immediate preoperative period is safe and the indicated practice in clean-contaminated procedures (other than elective colon resection) and in clean operative procedures associated with the use of foreign bodies (mesh, 
vascular grafts, etc.). Further study designs should strongly consider risk factors for individual patients when new antibiotic agents are tested or administration techniques are refined. A concentrated effort should be made in areas of clinical surgery, where the value of antibiotic prophylaxis has not been proven.

\section{REFERENCES}

1. Nichols RL. Surgical infections: prevention and treatment1965 to 1995. Am J Surg 1996;172:68-74.

2. Haley RW, Culver DH, Morgan WM, White JW, Emori TG, Hooton TM. Identifying patients at high risk of surgical wound infection. A simple multivariate index of patient susceptibility and wound contamination. Am J Epidemiol 1985;121:206-215.

3. Culver DH, Horan TC, Gaynes RP, Martone WJ, Jarvis WR, Emori TG, Banerjee SN, Edwards JR, Tolson JS, Henderson TS, Hughes JM, and the National Nosocomial Infections Surveillance System. Surgical wound infection rates by wound class, operative procedure, and patient risk index. Am J Med 1991;91(suppl 3B):152S-157S.

4. Nichols RL. Preventing surgical site infections: a surgeon's perspective. Emerg Infect Dis 2001;7:220-224.

5. Mangram AJ, Horan TC, Pearson ML, Silver LC, Jarvis WR. Guideline for prevention of surgical site infection, 1999. Hospital Infection Control Practices Advisory Committee. Infect Control Hosp Epidemiol 1999;20:250-278.

6 . Antimicrobial prophylaxis for surgery. Med Lett Drugs Ther 2004;(20):27-32.

7. Nichols RL, Condon RE. Antibiotic preparation of the colon: failure of commonly used regimens. Surg Clin North Am 1971;51:223-231.

8. Nichols RL, Smith JW, Garcia, RY, Waterman RS, Holmes JW. Current practices of preoperative bowel preparation among North American colorectal surgeons. Clin Infect Dis 1997;24:609-619.

9. Zmora O, Wexner SD, Hajjar L, Park T, Efron JE, Nogueras JJ, Weiss EG. Trends in preparation for colorectal surgery: survey of the members of the American Society of Colon and Rectal Surgeons. Am Surg 2003;69:150-154.

10. Nichols RL, Condon RE, Gorbach SL, Nyhus LM. Efficacy of preoperative antimicrobial preparation of the bowel. Ann Surg 1972;176:227-232.

11. Browder W, Smith JW, Vivoda LM, Nichols RL. Nonperforative appendicitis: a continuing surgical dilemma. J Infect Dis 1989;159:1088-1094.

12. Nichols RL, Smith JW, Klein DB, Trunkey DD, Cooper RH, Adinolfi MF, Mills J. Risk of infection after penetrating abdominal trauma. N Engl J Med 1984;311:1065-1070.

13. Nichols RL, Smith JW, Robertson GD, Muzik AC, Pearce P, Ozmen V, McSwain NE Jr, Flint LM. Prospective alterations in therapy for penetrating abdominal trauma. Arch Surg 1993;128:55-63; discussion 63-64.

14. Cant PJ, Smyth S, Smart DO. Antibiotic prophylaxis is indicated for chest stab wound requiring closed tube thoracostomy. Br J Surg 1993;80:464-466.

15. Nichols RL, Smith JW, Muzik AC, Love EJ, McSwain NE, Timberlake G, Flint LM. Preventive antibiotic usage in traumatic thoracic injuries requiring closed tube thoracostomy. Chest 1994;106:1493-1498. 